\title{
PUMVC3-hIGFBP-2 Multi-epitope Plasmid DNA Vaccine
}

National Cancer Institute

\section{Source}

National Cancer Institute. pUMVC3-hIGFBP-2 Multi-epitope Plasmid DNA Vaccine. NCI

Thesaurus. Code C95727.

A recombinant plasmid DNA vaccine containing mammalian expression vector, pUMVC3, encoding epitopes of human Insulin-Like Growth Factor-Binding Protein 2 (hIGFBP-2) with potential antineoplastic activity. Upon vaccination, pUMVC3-hIGFBP-2 multi-epitope plasmid DNA vaccine may produce IGFBP-2 that binds to cell surface, likely via integ rin complexes. This results in the dephosphorylation of the focal adhesion-kinase (FAK) and of the p42/44 MAP-kinases, thereby inactivating them; both kinases are involved in cell growth regulation. Furthermore, IGFBP-2 promotes de-adhesion of the cells in a dosedependent manner, hence impeding cell proliferation. The primary effect of IGFBPs appears to be modulation of IGF activity and control of IGF-mediated cell growth and metabolism. 